УДК 621.373 .5

\title{
ВЫСОКОЭФФЕКТИВНАЯ СХЕМА ГЕНЕРАТОРА ПОДКАЧКИ ЗАРЯДА С ПЕРЕКРЕСТНЫМИ СВЯЗЯМИ И ЧЕТЫРЫМЯ ТАКТОВЫМИ СИГНАЛАМИ*
}

\author{
МИНЛИН МА ${ }^{1,2}$, С. ЦАЙ ${ }^{1}$, Ц. ЦЗЯН ${ }^{1}$, И. СУН ${ }^{2}$ \\ ${ }^{1}$ Сянтаньский университет, \\ Китай, Сянтань, 411105, Хунань \\ ${ }^{2}$ Университет Хартфордшира, \\ Великобритания, Хатфилд, AL10 9AB
}

\begin{abstract}
Аннотация. Предложена монолитная схема генератора подкачки заряда с перекрестными обратными связями и четырьмя тактовыми сигналами для преобразователя постоянного напряжения в постоянное напряжение (DC-DC). При использовании указанной новой схемы тактовых сигналов такой преобразователь не имеет обратных потерь мощности и обладает уменьшенным напряжением пульсаций. Кроме того, максимальная разница напряжений между выводами всех транзисторов не превышает уровня напряжения питания, что позволяет решить проблему перенапряжения подзатворного оксида в традиционных схемах генератора подкачки заряда и повысить надежность. Предлагаемая схема генератора подкачки заряда не требует дополнительной схемы сдвига уровня, в результате чего коэффициент полезного действия увеличивается. Проведено моделирование предлагаемой схемы генератора подкачки заряда при использовании Spectre для моделирования 0,18 мкм КМОП-технологии фирмы TSMC. Результаты моделирования показали, что максимальный кпд преобразования напряжения для новой трехкаскадной схемы с перекрестными связями при входном напряжении 1,5 В составляет 99,8\%. Результаты сравнения традиционного и предлагаемого усовершенствованного генератора подкачки заряда дают существенное уменьшение пульсаций напряжения на выходе последнего.
\end{abstract}

Ключевые слова: генератор подкачки заряда с перекрестными связями; обратные потери мощности; напряжение пульсаций; четырехтактная схема

\section{1. ВВЕДЕНИЕ}

Схемы генераторов подкачки заряда с использованием заряда коммутируемых конденсаторов часто применяются для преобразования входного напряжения постоянного тока в выходное напряжение постоянного тока другого уровня (DC-DC). Такое устройство может формировать напряжение выше напряжения питания или ниже напряжения «земли». Гене- раторы подкачки заряда могут обеспечивать ток в десятки и сотни миллиампер для последующих блоков обработки сигнала. Подача стабильного повышенного напряжения постоянного тока на встроенные объекты интеллектуальной собственности (embedded Intellectual Properties) становится важной задачей.

Преимуществом схем подкачки заряда является их невысокая стоимость, низкий уро-

* Эта работа получила финансовую поддержку от Провинциального проекта по поддержке естественных наук, провинция Хунань (№ 2015JJ3126).

DOI: $10.20535 / S 002134701812004 \mathrm{X}$

() Минлин Ма, С. Цай, Ц. Цзян, И. Сун, 2018 


\section{БИБЛИОГРАФИЧЕСКИЙ СПИСОК}

1. Dickson, J. F. “On-chip high-voltage generation in MNOS integrated circuits using an improved voltage multiplier technique," IEEE J. Solid-State Circuits, Vol. 11, No. 3, p. 374-378, 1976. DOI: 10.1109/JSSC.1976. 1050739 .

2. Wu, J. T.; and Chang, K.-L. "MOS charge pumps for low-voltage operation," IEEE J. Solid-State Circuits, Vol. 33, No. 4, p. 592-597, 1998. DOI: 10.1109/ 4.663564.

3. Ker, M.-D.; Chen, S.-L.; and Tsai, C. S. "Design of charge pump circuit with consideration of gate-oxide reliability in low-voltage CMOS processes," IEEE $J$. Solid-State Circuits, Vol. 41, No. 5, p. 1100-1107, 2006. DOI: $10.1109 /$ JSSC.2006.872704.

4. Lee, H.; and Mok, P. K. T. "Switching noise and shoot-through current reduction techniques for switched-capacitor voltage doubler," IEEE J. Solid-State Circuits, Vol. 40, No. 5, p. 1136-1146, 2005. DOI: 10.11 09/JSSC.2005.845978.

5. Das, T.; Prasad, S.; Dam, S.; and Mandal, P. “A Pseudo Cross-Coupled Switch-Capacitor Based DC-DC Boost Converter for High Efficiency and High Power Density," IEEE Trans. Power Electronics, Vol. 29, No. 11, p. 5961-5974, 2014. DOI: 10.1109/TPEL.2014.2297 $\underline{972}$.

6. Kim, J.-Y.; Park, S.-J.; Kwon, K.-W.; Kong, B.-S.; Choi, J.-S.; and Jun, Y.-H. "CMOS charge pump with no reversion loss and enhanced drivability," IEEE Trans. Very Large Scale Integration Systems, Vol. 22, No. 6, p. 1441-1445, 2014. DOI: 10.1109/TVLSI.2013.2267214.

7. Mui, T. W.; Ho, M.; Mak, K. H.; Guo, J.; Chen, H.; and Leung, K. N. "An area-efficient 
96.5\%-peak-efficiency cross-coupled voltage doubler with minimum supply of $0.8 \mathrm{~V}$," IEEE Trans. Circuits
Systems II: Express Briefs, Vol. 61, No. 9, p. 656-660, 2014. DOI: $10.1109 /$ TCSII.2014.2331109. 